\title{
Quantum Structure Model of Judgement in Battlespace
}

\author{
Zhihua Song \\ Equipment Management and Safety Engineering \\ College, Air Force Engineering University, Xi'an, \\ China \\ szhele@163.com \\ Wanfang Che \\ Equipment Academy of Air Force, Beijing, China \\ lingling00@163.com
}

\author{
Han Zhang \\ Science College, Air Force Engineering University, \\ Xi'an, China \\ ola alo@126.com \\ Xiaobin Hui \\ Equipment Management and Safety Engineering \\ College, Air Force Engineering University, Xi'an, \\ China \\ cnilsw@126.com
}

\begin{abstract}
A quantum structure model of judgment in battlespace is presented and used to compute the probability of target identification and its evolution with incoming new evidence deprived from the refreshed reconnaissance image. On the one hand, human behavioral factors are hard to model using the classic probability theory in battlespace which is full of antagonism, uncertainty and irrationality. On the other hand, quantum theory is more general and powerful for representing and analyzing human's judgment and decisionmaking. In the task of identifying of real-false targets pair, a Mental Hamilton is constructed in order to transform a state to the next after new evidence arrived. The results computed on the basis of random-phase initial state of cognition and constructed mental Hamiltonian show that interference effect may appear and the Formula of Total Probability may be violated with quantum structure model of judgment, and these are just what usually happen in the human's judgment and decision-making in the reality.
\end{abstract}

Keywords-Quantum theory; Battlespace; Judgment

\section{INTRODUCTION}

Cognitive scientists have an influential research tradition for modeling cognitive processes with the classical probability framework. However, a large number of empirical phenomena, including conjunction fallacy, disjunction fallacy, unpacking effects and order effects on inference, have accumulated to the evidence that human judgment often goes directly against CP principles [3]. After finding the violations of $\mathrm{CP}$ principles in their influential program of research on judgment, Tversky and Kahneman have argued that heuristic and biases (such as representativeness, availability, and anchor adjustment) form the basis of human probability judgment under uncertainty [4]. The heuristic concept has had a massive influence on many subjects including psychology, management science and economics, culminating in a Nobel Prize award to Kahneman.

In recent years, there is a growing interest in the application of quantum theory to develop more coherent, comprehensive, and deductive theoretical explanations. These work formed an emerging field called Quantum
Cognition. Quantum cognition is concerned with applying the mathematical formalism of quantum theory, especially the quantum probability (QP) theory, to inspiring and formalizing computational models of various aspects of cognition such as information processing by human brain, decision-making, human memory, concepts and conceptual reasoning, human judgment, and perception. Many wellestablished empirical findings have natural and straightforward explanations with QP principles while they are hard to reconcile with $\mathrm{CP}$ principles [5]. The main characteristics of QP theory (order/context effects, interference, superposition, entanglement), provide us with lots of advantages in understanding cognitive process and decision-making. QP provides a promising framework for modeling human decision-making [6]. It may also provide us with an approach to model the judgment and decisionmaking in the cognitive domain of warfare.

Sun Tzu said: If you know the enemy and know yourself, your victory will not stand in doubt. This means that Information superiority and decision superiority [7] are at the core of every military operation. Military leaders must know the enemy to acquire Information superiority. Then military leaders must be able to know the advantages and disadvantages of their force correctly in order to translate the information superiority into superior knowledge and decisions. Fig. 1 shows the translation process through the three domains of warfare. 


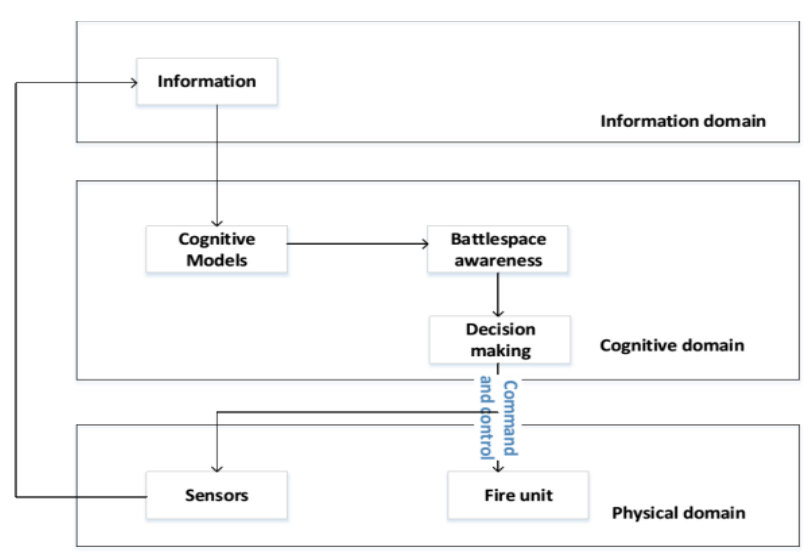

Figure 1. Three domains of warfare and the translation process from information to decision

In the battlespace, information superiority never means perfect or exact information because of the existence of the fog of war. Thus real judgments and decisions usually made in the state of indeterminacy [8] and based on beliefs concerning the likelihood of uncertain events, such as the identification of a missile launch vehicle in satellite imagery, the damage degree evaluation of airport runway after strike. These beliefs may be sensitive to the environment and have an interference effect. Quantum theory could provide an appropriate mathematical framework for understanding and modeling certain behavioral aspects of cognition domain of warfare. A better understanding of human behavioral factors is the foundation of innovation of the organization and doctrine on one side and making better predictions about the enemy to take countermeasures on the other side.

\section{QUANTUM MODEL OF JUDGMENT IN BATTLESPACE}

State representation. To model judgment in the cognition domain of warfare, we will postulate a set of basis states. The basis states are used to represent the various mental states about preference orders over judgment, and can be measured during an operation. Accordingly, they can be represented by basis vectors of a vector space.

$$
\Omega=\{|1\rangle,|2\rangle, \cdots,|m\rangle\}
$$

According to a quantum process, an individual experiences a superposition of all these states, and at any moment the person remains uncommitted to any specific state [6]. A superposition state of the mental at any moment in time $t$ is defined as a linear combination of the basis vectors in $\Omega$ :

$$
|\psi(t)\rangle=\sum_{j=1}^{m}\langle j|\psi(t)| j\rangle=\sum_{j=1}^{m} \psi_{j}(t)|j\rangle
$$

Where $\psi_{j}(t)$ is the probability amplitude of observing the basis state $|j\rangle$ at time $t$.

State transitions. A quantum state can be transformed into another state. The probability amplitude of starting in state $|\psi\rangle$ and then observing basis state $|i\rangle$ can be expressed in terms of the $\Omega$ basis as

$$
\langle i|U| \psi\rangle=\sum_{j=1}^{m}\langle i|U| j\rangle\langle j \mid \psi\rangle=\sum_{j=1}^{m} u_{i j}\langle j \mid \psi\rangle
$$

Where $u_{i j}=\langle i|U| j\rangle$ is the probability amplitude of transiting from basis state $|j\rangle$ to basis state $|i\rangle$ going through the unitary operator $U$.

Then the initial state $|\psi(0)\rangle$ is processed by the unitary operator $U$ for some period of time $t$, then it produces the new state

$$
|\psi(t)\rangle=U|\psi(0)\rangle
$$

Time evolution of probability distributions. The time evolution of the superposition state $|\psi\rangle$ is described by the schrödinger equation

$$
i \frac{d}{d t}|\psi(t)\rangle=H|\psi(t)\rangle
$$

The Hamiltonian $H$ has elements $h_{i j}=\lim _{\tau \rightarrow 0}\left(u_{i j}(\tau)-\right.$ $\left.u_{i j}(0)\right) / \tau$ in row $i$ column, representing the instantaneous rate of change from $|j\rangle$ to $|i\rangle$. The solution to the schrödinger equation is given by the matrix exponential of the Hamiltonian matrix:

$$
|\psi(t)\rangle=e^{-i H t}|\psi(0)\rangle
$$

The probability for for each state is obtained by taking the squared magnitude of the corresponding probability amplitude:

$$
P_{j}(t)=\left.\left|\psi_{j}(t)\right\rangle\right|^{2}
$$

\section{TARGET IDENTIFICATION COGNITIVE EVOLUTION MODEL}

In the task of image interpretation, a human interpreter derives useful information from images of various surveillance and reconnaissance platforms in order to identify objects and judge their significance. Target identification is the premise for tactical decision making and weapon-target assignment optimization. Sometimes one must identify the decoys from the real target (e.g., a missile launch unit). Mistake the decoys for real targets could lead to a wasted fire or fall into the enemy's trap, and so could the error of misidentifying a real foe as a decoy. However, warfare will always be full of antagonism and uncertainty. Having a picture of what is happening reduces uncertainty about the target while the enemy will certainly have a strong wish to raise this uncertainty by take some countermeasures. Thus the confidence rating of the image interpreter associate with target identification may be fluctuated by contradicting evidences derived from the discretely reached images.

\section{A. Initial state}

Basis states. The basis states represent all the possible identification outcomes with different confidence rating measures. A binary choice (false versus real) is represented by two orthonormal basis states, $|-\rangle$ nd $|+\rangle$ for false target and real target respectively. The confidence rating is 
represented by $m$ orthonormal basis states $|0\rangle,|1\rangle, \ldots, \mid+(m-$ $1)\rangle$. Thus the tensor product of these two basis states forms a complete set of basis states

$$
\Omega_{I}=\{|-(m-1)\rangle,|-m\rangle, \cdots,|+m\rangle,|+(m-1)\rangle\}
$$

Then a superposition state is a linear combination of these basis states.

$$
|\psi\rangle=\sum_{j=-(m-1)}^{m-1} \psi_{j}|j\rangle
$$

Where $\psi_{j}(t)$ denotes one has $|j|$ confidence rating of identifying a target as real (if $j>0$ ), false (if $j<0$ ), or unknown $(j=0)$ with probability amplitude of $\psi_{j}$.

Task definition. Suppose there are two targets A and B, one of which is the real target and the other is the decoy . Before the arriving of the first image, there is no prior information available to identify the potential target from its decoy. The target identification process begins with an initial state with even distribution of probability.

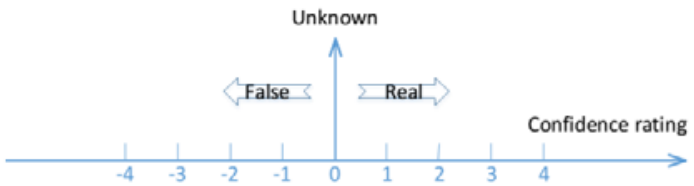

Figure 2. Basis states of identifying a target

We set that the initial probability of each basis state is equal, and the initial superposition state is a linear combination of these basis states.

$$
\begin{aligned}
\left|\psi_{A}(0)\right\rangle & =\sum_{j=-(m-1)}^{m-1} \psi_{A j}|j\rangle \\
\left|\psi_{B}(0)\right\rangle & =\sum_{j=-(m-1)}^{m-1} \psi_{B j}|j\rangle
\end{aligned}
$$

Then in the task of distinguishing them together, the basis states of the task in one's mind would be the same as $\Omega_{\mathrm{I}}$, and $\psi_{j}$ represents that one has $6|j|$ confidence rating of identifying $\mathrm{A}$ as real and $\mathrm{B}$ as false (if $j>0$ ), $\mathrm{A}$ false and $\mathrm{B}$ real $(j<0)$, or unknown $(j=0)$ with probability amplitude of $\psi_{j}(0)=\psi_{A j}(0)+\psi_{B j}(0)$.

$$
|\psi(0)\rangle=\sum_{j=-(m-1)}^{m-1}\left(\psi_{A j}(0)+\psi_{B j}(0)\right)|j\rangle
$$

To illustrate the similarity between the model and human cognition process in identifying the targets together, it is helpful to consider an example. We set $m=20$ and the argument of each basis state was set to be random as shown in the first row insets of Fig.4 The probability of identifying $A$ as real $(j>0)$, false $(j<0)$, or unknown $(j=0)$ is the squared magnitude of the probability amplitude $\psi_{A j}(0)$, see Fig.4.

$$
\begin{aligned}
& P\left(A_{j}\right)=\left|\psi_{A j}(0)\right|^{2}=1 /(2 m+1)=1 / 41 \\
& P\left(B_{j}\right)=\left|\psi_{B j}(0)\right|^{2}=1 /(2 m+1)=1 / 41
\end{aligned}
$$

The probability of identifying $A$ as real and $\mathrm{B}$ as false $(j>$ $0), A$ false and $B$ real $(j<0)$, or unknown $(j=0)$ is the squared magnitude of the probability amplitude $\psi(0)$, see Fig.4.

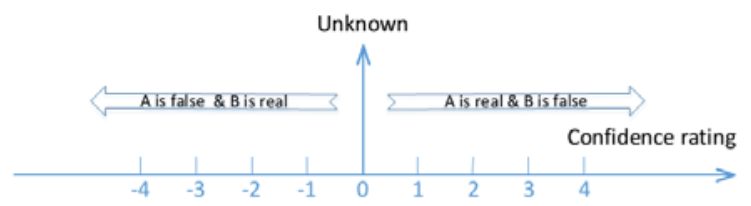

Figure 3. Basis states of identifying a pair of targets A and B together

$$
P\left(A_{j} B_{-j}\right)=P\left(A_{j}\right)+P\left(B_{j}\right)+2 R e\left(\psi_{A j}\right) \psi_{B-j}
$$

Where the term $2 \operatorname{Re}\left(\psi_{A j}\right) \psi_{B-j}$ was called the Interference item. When the interference item doesn't equal to zero, we say there is an interference effect in the judgment.

With the results in Fig.4, we can see that it's apparently that there exists an interference effect when identifying A and B together (See last inset in the second row of Fig.4). With this effect, the judgment of human will no longer be even distributed. The final result may be sensitive to the environment, context and even worthless information.

\section{B. Evolution states}

Then we assume that new information about these two targets is derived from the discretely refreshed image by image interpreters. The information provides some evidence about the target's attributes (real or false) and changes the initial state $|\psi(0)\rangle$ to a new state $|\psi(1)\rangle$ which represents the probability amplitude distribution over basis states (Here we set $t=1$ ).

$$
|\psi(1)\rangle=\mathrm{e}^{-\mathrm{iH}}|\psi(0)\rangle
$$

We construct a Mental Hamiltonian $H$ of this transition as (15), which simulate an interpreter's cognitive process. After reading the image, the contrary basis states on the same confidence rating may collide with each other and new confidence rating arise from the middle state because of the new evidence of identifying the targets pair.

$$
h_{k l}= \begin{cases}\varepsilon_{k-m}+0.5\left(\pi-\left|\varepsilon_{k-m}-\varepsilon_{m-k}\right|\right) & \text { if } \varepsilon_{k-m}>\varepsilon_{m-k} \& l \neq\lfloor 0.5 m\rfloor+1 \\ \varepsilon_{m-k}-0.5\left(\pi-\left|\varepsilon_{k-m}-\varepsilon_{m-k}\right|\right) & \text { if } \varepsilon_{k-m} \leq \varepsilon_{m-k} \& l \neq\lfloor 0.5 m\rfloor+1 \\ \varepsilon_{k-m}-\varepsilon_{0}+i \cdot \log (r) & \text { if } l=\lfloor 0.5 m\rfloor+1\end{cases}
$$

Where $\varepsilon_{k-m}$ is the argument of complex number $\psi_{k-m} . r$ is the enabling function to increase or decrease the confidence rating. 

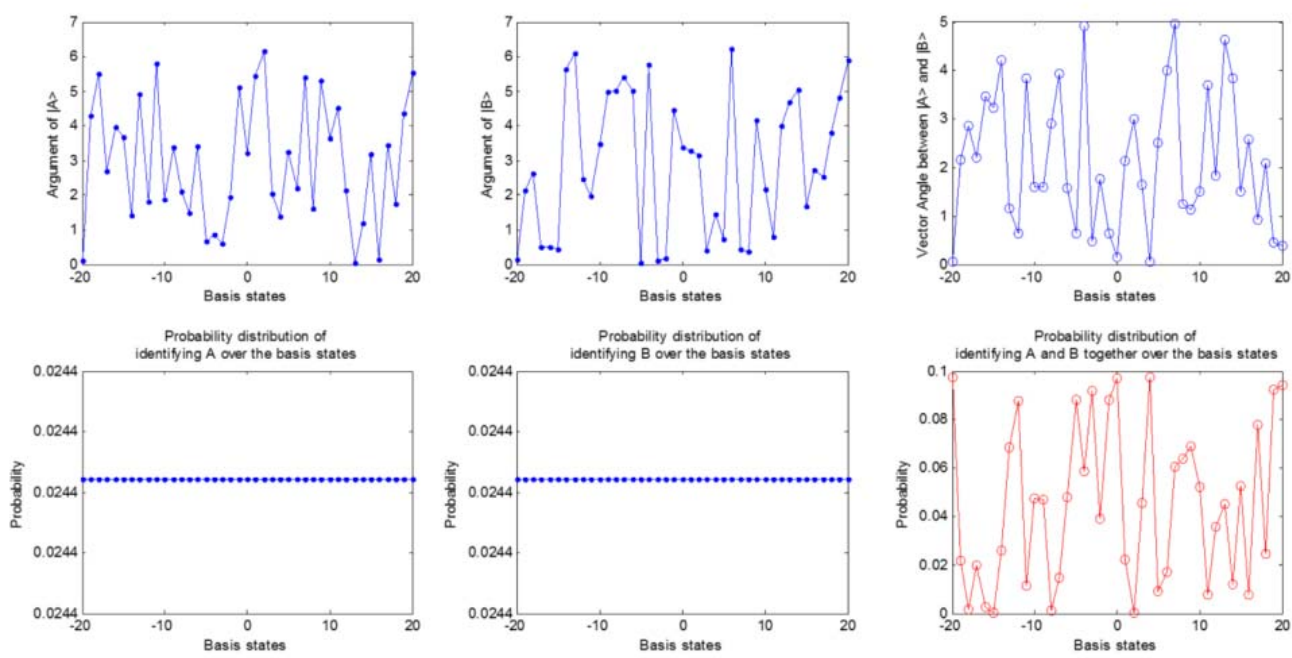

Figure 4. The argument of state is random and the probability of identifying each target over basis states is even distributed. An interference effect appeared when identifying A and B together.
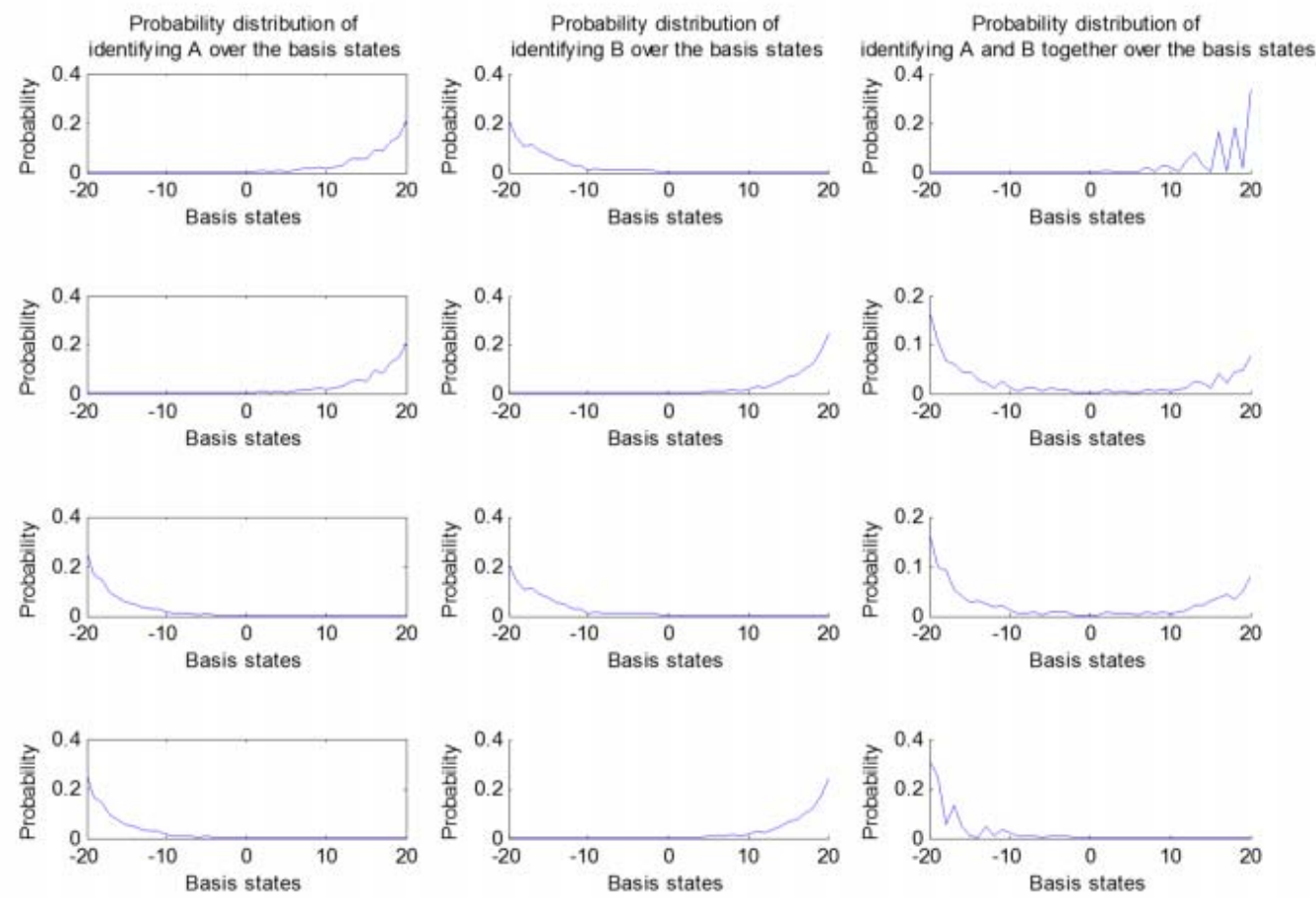

Figure 5. Different types of mental hamilton(i.e., Real Confirming Mental Hamiltonian and False Confirming Mental Hamiltonian) lead to different types of transition of probability distribution over basis states.

We call the Mental Hamilton (i.e Real Confirming Mental Hamiltonian when new image gives the interpreter an increase in his or her $|+\rangle$ state of judgment (e.g., first inset in the first row in Fig.5), and we call it is a False
Confirming Mental Hamiltonian while the effect of transition is an increase in $|-\rangle$ state of judgment (e.g., second inset in the first row in Fig.5). 
If the event $\mathrm{A}^{+}\left(\mathrm{A}^{-}\right)$was defined as identifying $\mathrm{A}$ as real (false) over various confidence ratings, then the probability of $\mathrm{A}^{+}\left(\mathrm{A}^{-}\right)$is as follows

$$
\left\{\begin{array}{c}
P\left(A^{+}\right)=\sum_{j=1}^{m-1}\left|\psi_{j}\right|^{2}+0.5\left|\psi_{0}\right|^{2} \\
P\left(A^{-}\right)=\sum_{j=-(m-1)}^{-1}\left|\psi_{j}\right|^{2}+0.5\left|\psi_{0}\right|^{2}
\end{array}\right.
$$

Where we divided the $\left|\psi_{0}\right|^{2}$ equally into $\mathrm{P}\left(\mathrm{A}^{+}\right)$and $\mathrm{P}\left(\mathrm{A}^{-}\right)$for simplicity.

Then according to the data in Fig.5, the probabilities of these events are computed and shown in Table.1.

TABLE I. PROBABILITIES OF VARIOUS TARGET IDENTIFICATION RESULTS

\begin{tabular}{lcccccc}
\hline & $P\left(A^{+}\right)$ & $P\left(A^{-}\right)$ & $P\left(B^{+}\right)$ & $P\left(B^{-}\right)$ & $P\left(A^{+} B^{-}\right)$ & $P\left(A^{-} B^{+}\right)$ \\
\hline test1 & 0.9765 & 0.0235 & 0.0250 & 0.9750 & 0.9629 & 0.0371 \\
test2 & 0.9765 & 0.0235 & 0.9848 & 0.0152 & 0.3531 & 0.6469 \\
test3 & 0.0116 & 0.9884 & 0.0250 & 0.9750 & 0.3779 & 0.6221 \\
test4 & 0.0116 & 0.9884 & 0.9848 & 0.0152 & 0.0167 & 0.9833 \\
\hline
\end{tabular}

According to the task definition, if the interpreter's state $\psi(1)$ about $A$ finally collapsed onto a state representing " $A$ is real", then his judgment about $B$ would be " $B$ is false" by default. The probability of event $\mathrm{B}^{-}$under the condition of " $A$ is real" equals to 1 .

Similarly, the probability of event $\mathrm{B}^{-}$under the condition of " $A$ is false" equals to 0 .

$$
P\left(B^{-} \mid A^{-}\right)=0, P\left(B^{-} \mid A^{+}\right)=1
$$

If we compute the probability of $\mathrm{B}^{-}$using the formula of total probability, we will have

$\mathrm{P}\left(\mathrm{B}^{-}\right)=\mathrm{P}\left(\mathrm{B}^{-} \mid \mathrm{A}^{-}\right) \mathrm{P}\left(\mathrm{A}^{-}\right)+\mathrm{P}\left(\mathrm{B}^{-} \mid \mathrm{A}^{+}\right) \mathrm{P}\left(\mathrm{A}^{+}\right)=\mathrm{P}\left(\mathrm{A}^{+}\right)$

It is apparently that $(15)$ is not true for the data in Table.1. So the results in Fig.5 violate the formula of total probability.

The probability of the event " $\mathrm{A}$ is real" is just a judgment of an image interpreter. It represents the strength with which one believes the event will happen and it is based on ones belief degree that equals between 0 and 1 . After the judgment is revealed the superposition state will collapse onto exactly one basis state. A well-trained image interpreters belief degree is close to the truth and usually has a high probability of collapse onto the correct answer state. A beginner may be confused by the elements derived from the image and usually has a lower probability of collapse onto the correct answer state.

\section{CONCLUSIONS}

The general purpose of this study is to provide a more general and humanlike model of judgment in battlespace. Firstly, a general quantum structure model of judgment in battlespace is developed on the basis of the quantum theory. Secondly, we developed a specific application of the quantum structure model in the task of target identification. Its initial state is assumed to be random-phased and a Mental Hamilton is constructed to simulate the evolution of interpreter's mind. The derived probability distribution over basis states show that the model has very different attributes compared with classic model. There exist an interference effect and the formula of total probability may be violated. It sounds strange but it's usual in human judgment and decision-making. Quantum structure model based on the quantum theory provides a more human-like approach to describe the information processing and judgment of human. It provides a promising new way to model judgment in battlespace.

\section{REFERENCES}

[1] Griffiths, T. L., \&Chater, N., Kemp, C., Perfors, A. Tenenbaum, J. B. (2010) Probabilistic models of cognition: Exploring representations and inductive biases. Trends in Cognitive Sciences 14:357-64.

[2] Gilovich, T., Griffin, D., \&Kahneman, D. (2002). Heuristics and biases: The psychology of intuitive judgment (pp. 49-81): New York, NY: Cambridge University Press.

[3] Busemeyer, J.R., Wang Z, Townsend JT (2006) Quantum dynamics of human decision-making. Journal of Mathematical Psychology. 50(3):220-241

[4] Tversky, A.,\&Kahneman, D. (1974) Judgment under uncertainty: Heuristics and biases. Science 185:1124-31.

[5] Pothos, E.M., \&Busemeyer, J.R.(2013). Can quantum probability provide a new direction for cognitive modeling? Behavioral and brain sciences .36:255-328.

[6] Pothos, E.M., \&Busemeyer, J.R. (2009). A Quantum Explanation for Violations of Rational Decision Making. Proceedings of the Royal Society B.

[7] Alberts, D.S., Garstka, J.J., \& Stein, F.P. (1999). Network centric warfare: Developing and leveraging information superiority.

[8] Liu, B. (2012a). Uncertainty theory (4th ed.). Uncertainty Theory Laboratory. Available from: http://orsc.edu.cn/liu/ut.pdf. 